Annales Geophysicae (2004) 22: 3805-3814

SRef-ID: $1432-0576 / \mathrm{ag} / 2004-22-3805$

(C) European Geosciences Union 2004

\title{
Radar meteor decay rate variability and atmospheric consequences
}

\author{
W. K. Hocking \\ University of Western Ontario, London, Ontario, Canada \\ Received: 16 December 2003 - Revised: 11 July 2004 - Accepted: 25 October 2004 - Published: 29 November 2004 \\ Part of Special Issue "10th International Workshop on Technical and Scientific Aspects of MST Radar (MST10)"
}

\begin{abstract}
The reasons for scatter in plots of $\log$ (inverse decay times) vs. height for radio meteor echoes are examined, and an explanation for the characteristics is offered. Effects like temperature variability, pressure variation, angular detection accuracy, pulse length, phase errors, plasma processes and variation in meteoroid metallic content are considered. Using computer simulations the observed scatter is reproduced to good accuracy, and then these results are utilized to develop a new procedure that can be used to determine temperatures in the meteor region. These same studies also permit determination of some limited information about the nature of the diffusive expansion process and the variability in the metallic content of meteors. The impact of the quality of phase calibration of interferometric radars on accurate reproduction of atmospheric temperatures is also examined.
\end{abstract}

Key words. Electromagnetics (scattering and diffraction) Meteorology and atmospheric dynamics (instruments and techniques) - Radio science (interferometry)

\section{Introduction}

When meteors enter the Earth's atmosphere, they produce a plasma trail from which radio waves may reflect. The amplitude of the radar signal received at the ground shows a rapid increase as the trail forms, then decays in time. For the most common meteors, the so-called "underdense meteors", the temporal variation in amplitude varies according to

$A=A_{0} e^{-\left(16 \pi^{2} D_{a} t\right) / \lambda^{2}}=A_{0} e^{-\ell n 2 \frac{t}{\tau_{1 / 2}}}$,

where $t$ is time, $\lambda$ is the radar wavelength, $D_{a}$ is the "ambipolar diffusion coefficient", and $\tau_{1 / 2}$ is the time for the amplitude to fall to one-half of its maximum value. $A(t)$ is the received field strength at time $t$, with $t=0$ being the time at which the meteor signal reaches its peak amplitude, just prior to the onset of decay. Typical half-amplitude decay times $\tau_{1 / 2}$ are of the order of 0.01 to $0.3 \mathrm{~s}$ for a radar operating at a frequency in the range 30 to $50 \mathrm{MHz}$. (e.g. see Hocking et al., 1997, and references therein). Collective application of decay times from many meteors may be used to determine temperatures at (typically) $90 \mathrm{~km}$ altitude. The concept behind this process has been known for many years (e.g. see Jones, 1975, and references therein), but routine and continuous application of the procedure had to wait until the 1990's, when diffusion rates were better quantified (e.g. Jones and Jones, 1990), and when faster computers permitted better discrimination of meteor echoes from other types of impulsive interference (e.g. Hocking et al., 2001a). Tsusumi (1994) examined relative temperaure perturbations, and then Hocking et al. (1997) determined absolute temperatures. However, the latter work was encumbered by a need to know the atmospheric pressures, and the CIRA pressures used at the time were generally unreliable. Therefore, Hocking (1999) developed an alternative method which employed the mean slope determined from the graphs of the $\log$ (inverse decay time) vs. height. This method produced temperatures which were independent of any need for assumptions about the atmospheric pressure.

The above theory assumes that the expansion process occurs in a region where the effects of electric and magnetic fields are damped by collision with the neutrals, but at high enough altitudes, usually above $93 \mathrm{~km}$, expansion can be anisotropic (e.g. see Cervera and Reid, 2000). Theory relating to this effect has been presented by a variety of authors, including most recently Robson (2001), Oppenheim et al. (2000) and Dyrud et al. (2001, 2002). Most of the data used here apply to heights below $93 \mathrm{~km}$, and so the above theories do not affect the results significantly - indeed less than $8 \%$ of meteors used in this study lie above $95 \mathrm{~km}$ altitude. Nevertheless, for completeness the effects of magnetic and electric fields will also be considered later in this document. 


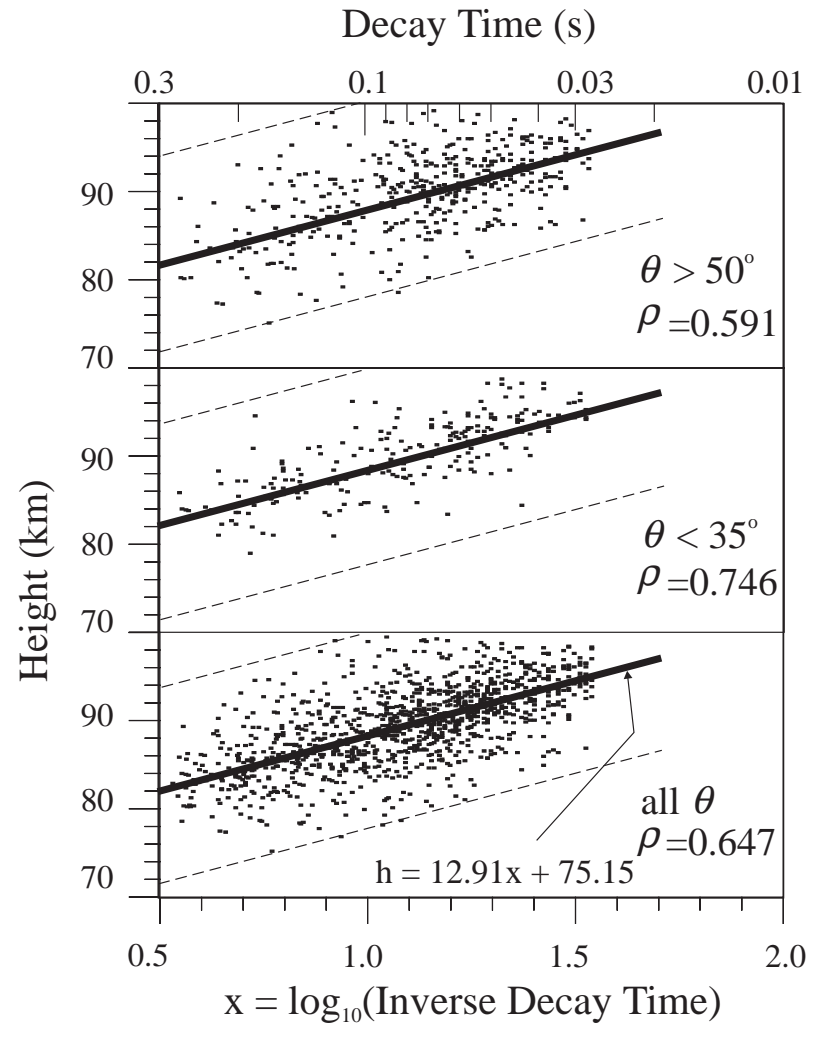

Fig. 1. Typical plots of $\log _{10}$ (inverse decay time) as a function of height for (a) meteors with zenith angles between $50^{\circ}$ and $70^{\circ}$, (b) zenith angles less than $35^{\circ}$, and (c) zenith angles between $0^{\circ}$ and $70^{\circ}$.

Previous studies of meteor decay times as a function of height (Hocking et al., 1997; Hocking, 1999; Cervera and Reid, 2000) have concentrated on variations of the mean values as a function of altitude, or have used fitting of best-fit polynomials (or even linear functions) to the data. However, plots of $\log$ (inverse decay time) as a function of height show considerable fluctuation and scatter, and the correlation coefficient of the log of the inverse decay time vs. height is typically in the range 0.45 to 0.75 after application of suitable outlier rejection (Hocking, 1999; Cervera and Reid, 2000). In order to optimally employ these data, it is important to understand the causes of this statistical scatter. Better understanding can help us to design better radars, and can also give us insights into the natural variability of related atmospheric and astronomical variables. Fitting of curves or straight lines to data with scatter can also be biased by the relative errors of the ordinate and abscissa, as shown by Hocking et al. (2001b). Better understanding of the causes of the scatter can also help in interpretation of such fitting algorithms.

To begin, some typical real data recorded by interferometric meteor radars are presented. Dependencies of the decaytime correlation coefficient on temperature are then seen at several sites from polar to mid latitudes and in both hemispheres. This prompts development of a numerical model to simulate the expected effects. By adjusting a limited num- ber of parameters it is possible to produce good agreement with observations, and the values of these parameters then give new insight into the plasma-trail formation process as well as the detection process. In particular, it is possible to place limits on some key atmospheric and astronomical parameters.

\section{Instrumentation}

A variety of interferometric radars are used in these studies, but all are based around the design of the SKiYMET radar (Hocking et al., 2001a). This is an interferometric radar which utilizes a transmitter and 5 separate receiving antennas to locate meteors in the sky. Pulses of radio waves are transmitted at high pulse repetition frequencies (typically 1000 to $2500 \mathrm{~Hz}$ ) and signals are received separately on the 5 receiving antennas. Each antenna then feeds signal directly into one of 5 receivers, and in-phase and quadrature components are digitized for each receiver. By comparing the phases of the received signals, meteor trail locations can be found to an accuracy of typically $1.5^{\circ}$ (e.g. Jones et al., 1998). The radars used in this study are located at Socorro, New Mexico $\left(34.1^{\circ} \mathrm{N}, 106.9^{\circ} \mathrm{W}\right)$, Resolute Bay $\left(75^{\circ} \mathrm{N}, 95^{\circ} \mathrm{W}\right)$, Learmonth Australia $\left(22.2^{\circ} \mathrm{S}, 114.1^{\circ} \mathrm{E}\right)$ and Delamere Australia $\left(35.5^{\circ} \mathrm{S}, 138.1^{\circ} \mathrm{E}\right)$ and at London (Ont), Canada (43N, $81 \mathrm{~W})$. Results have been checked against other SKiYMET radars situated elsewhere. The principal radar used is that at Resolute Bay, because the polar regions are the locations where the deepest range of temperatures is known to occur (e.g. Lübken, 1999), allowing for the best test situation for the models. More details about the specific operation of the SKiYMET radars can be found in Hocking et al. (2001a) and Hocking et al. (2001c).

\section{Observations}

Figure 1 shows typical scatter plots of $\log$ (inverse decay time) vs. height, taken with the Socorro radar, with meteors grouped according to zenith angle. Only meteors that satisfy the extensive criteria outlined by Hocking et al. (2001a) (primarily based on decay rate characteristics) are accepted, which largely restrict the accepted meteors to underdense meteors. The tests are not absolute in this regard, since a truly underdense meteor requires a line-density (i.e. the plsama density per unit length along the meteor trail) of less than $10^{14} \mathrm{~m}^{-1}$, and the actual line density is not normally measured with these radars. However, these tests are sufficient enough to ensure that by far the majority of meteors accepted satisfy the conditions of being underdense, or are very close to it. Additional tests which further ensure selection of under-dense echoes are also discussed in the next paragraph. No discrimination in regard to meteor trail alignment with respect to the magnetic field $B$ has been considered at this time. There have been some simulations that suggest care is needed when meteor trails are aligned with the magnetic field (e.g. Kaiser, 1969), but even if this were a problem, such meteors 
represent only a very small percentage of the whole sample. The simulations of Dyrud et al. $(2001,2002)$ show that magnetic field and plasma processes are most important above about $95 \mathrm{~km}$, which is above the height of maximum meteor detection for all the radars used in these studies. Since the analysis herein concentrates on underdense meteors primarily below $93 \mathrm{~km}$ altitude, it appears that plasma processes should not seriously affect our results. Nevertheless, in order to be cautious, we do present a separate study of decay times at the greater heights in Sect. 8, and this confirms these expectations.

The data shown in Fig. 1 were taken on 14 September 2002, and represent sample data from that day. The upper graph shows cases where meteors with zenith angles greater than $50^{\circ}$ and less than $70^{\circ}$ were chosen, while the middle graph shows cases where zenith angles less than $35^{\circ}$ are used. The lower graph shows the case when all data are used out to $70^{\circ}$. Not all data for the day have been used since there are so many points that they overlap and the graphs become harder to interpret. Therefore, approximately $1000 \mathrm{me}-$ teors have been randomly selected for this demonstration, although much more extensive analyses using much larger data sets were carried out to confirm the results presented. Data outside the broken lines were rejected as outliers; these were few in number, but arose due to a few echoes from overdense meteor and impulsive spatially coherent RF interference, and can adversely affect the analyses if not rejected. (As an example, a simple average of the decay times at a height of $95 \mathrm{~km}$ without this rejection produces an unrealistically large mean value because a single overdense echo with a decay time of, say, $0.3 \mathrm{~s}$ has the same effect as 10 meteors with decay times of around $0.03 \mathrm{~s}$. Such effects are mitigated by averaging in logarithmic coordinates for inverse decay times.)

The most important result here is that the zero-lag correlation coefficient (also called the regression coeffient) of $\log$ (inverse decay time) vs. height is clearly greater when smaller zenith angles are chosen. However, it should also be noted that the best-fit lines (solid sloping lines) show that there is little to no variation in slope and offset as a function of zenith angle. In fact, the best-fit line varies in slope by only $2 \%$ between the different cases, being 12.7 for the upper case and 13.03 for the second case, even though the regression coefficient varies by over 0.15 . This would lead to an error of less than $4 \mathrm{~K}$ in temperature if these data were used to calculate temperatures. The offset of the best-fit line from zero is similarly almost unchanged between figures. Similar internal self-consistency is obtained at other sites, and at other times of the year. These sites include stations as far north as Resolute Bay, and near-equatorial sites. However, while the mean characteristics (slope and offset) are almost unchanged, without fail the correlation is worse when larger zenith angles are used. This is the first key point to be made. This is further dealt with below.

It is now appropriate to turn to seasonal variations. Decaytime correlation coefficients have been determined just as in Fig. 1 for a variety of sites. In general, data covering typically 2 to 4 days were used for each determination. At all sites the

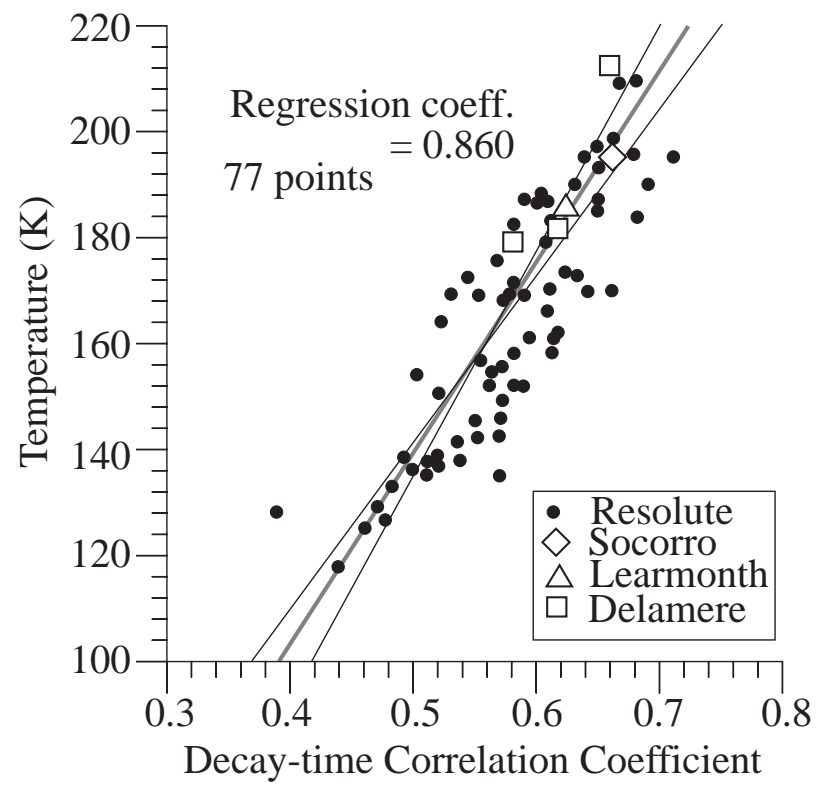

Fig. 2. Plot of the decay-time correlation coefficient (deduced from graphs like Fig. 1c) as a function of true temperature, for various sites in both the Northern and Southern Hemisphere. Best-fit lines show the regression of the ordinate on the abscissa (shallowest slope), the regression of the abscissa on the ordinate (steepest line) and the best-fit line assuming equal relative errors. The regression coefficient is shown.

correlation coefficient decreases at lower temperatures. For example, at Resolute Bay the temperature varies from about $220 \mathrm{~K}$ in winter to about $130 \mathrm{~K}$ in summer. Figure 2 shows a plot of the regression coefficient as a function of temperature, where the black filled circles were obtained using 4-day data groups at Resolute Bay, from 1 January to 30 June 2001. The temperature data have been deduced from the radar but were also confirmed to be reasonable by comparison with Lübken and von Zahn (1991) and Lübken (1999). Also added to the same plot are data from the SKiYMET radar at Socorro in New Mexico (previously at Starfire - e.g. see Liu et al., 2002; Hocking and Hocking, 2002), and the Genesis radar in Australia, both during its normal location at Delamere $\left(35.5^{\circ} \mathrm{S}\right)$ and during a campaign at Learmonth $\left(22.2^{\circ} \mathrm{S}\right)$ (Fuller, private communication). More data are available from all these sites, but the point here is simply to illustrate that the Resolute Bay are not atypical. The Resolute Bay data represent our main data group because of the wide range of temperatures available at that site.

Also shown on the graph are least-squares straight-line fits of the temperature as a function of decay-time correlation coefficient, first assuming zero error in the abscissa (shallowest line), then assuming zero error in the ordinate (steepest line). The middle line was deduced assuming approximately equal relative error in each variable.

It is clear that the decay-time correlation coefficient and the temperature are approximately linearly related (with some scatter), and the relationship is largely site independent. 
The reason for this needs to be addressed. It could be proposed that a reduced correlation coefficient might artificially lower the temperature by biasing the best-fit line, but it has already been seen in Fig. 1 that this does not occur to any significant degree, provided that the phases of the radar antennas are properly calibrated and the echo heights are accurate. This suggests that there is a geophysical basis for the correlation, and that the variation in decay-time correlation coefficient is a seasonal effect, or is directly related to temperature. Alternatively, it could in fact be an astronomical effect relating to the character of the meteors and the Earth's position in orbit. However, the astronomical hypothesis can easily be dismissed because Fig. 2 includes Southern Hemisphere data, which should have a reversed seasonal dependence to Northern Hemisphere data if the effect were dependent on the position of the Earth in its orbit. Therefore, it is proposed that either cooler temperatures lead to repressed correlation coefficients, or some alternate seasonal dependence produces the effect. The purpose of the next section of this paper is to examine the possible reasons for this effect in more detail.

\section{Causes of errors in $\tau_{1 / 2}$}

In this section, an attempt is made to reproduce the scatter shown in Fig. 1 and also the dependence of the correlation on temperature.

The expression for the decay time of radio echoes from underdense meteor trails is

$\tau_{1 / 2}=\frac{\ln 2 \lambda^{2}}{16 \pi^{2} D_{a}}$,

where

$D_{a}=K_{a m b} \frac{T^{2}}{P}$

and where $K_{a m b}$ is a constant. Specific details about $K_{a m b}$ have been outlined in Jones and Jones (1990), Chilson et al. (1996), and Hocking et al. (1997). It is proportional to the zero field reduced mobility.

It should be noted that while some effort has been made to accept predominantly underdense meteors, the requirement that all meteors be underdense is not critical. The primary condition is that the meteors that are accepted have decay characteristics which satisfy Eqs. (1) and (3), and Hocking (1999) has shown that this is generally true for the criteria outlined by Hocking et al. (2001a).

Combining Eqs. (2) and (3) gives

$$
\begin{aligned}
& \log _{10}\left(\frac{1}{\tau_{1 / 2}}\right)= \\
& \log _{10}\left(\frac{16 \pi^{2}}{\ln 2 \lambda^{2}}\right)+\log _{10}\left(K_{a m b}\right)+2 \log _{10} T-\log _{10} P .
\end{aligned}
$$

The most successful method for determining meteor temperatures utilizes the fact that $\log _{10}\left(\frac{1}{\tau_{1 / 2}}\right)$ depends on $\log _{10} P$, and that $P$, in turn, varies with height according to the scale height, which, in turn, depends on temperature. Various additional corrections are required as outlined by Hocking (1999).

The error in $\log _{10}\left(\frac{1}{\tau_{1 / 2}}\right)$ is given by

$$
\begin{aligned}
& \Delta\left(\log _{10}\left(\frac{1}{\tau_{1 / 2}}\right)\right)= \\
& \frac{1}{\ln 10} \sqrt{\left[\frac{\Delta K_{a m b}}{K_{a m b}}\right]^{2}+\left[\frac{\Delta P}{P}\right]^{2}+4\left[\frac{\Delta T}{T}\right]^{2}} .
\end{aligned}
$$

\section{Temperature, $K_{a m b}$ and pressure-related errors}

Variability in $\log _{10}\left(1 / \tau_{1 / 2}\right)$ can therefore arise due to variability in temperature, variability in pressure or variability in $K_{a m b}$, which itself varies according to the alkali and metallic constituents of the meteor trail and also the chemical composition (e.g. oxygen concentration) of the atmosphere. It is assumed that the latter composition does not change substantially throughout the year, but changes to the heights at which meteors ablate could alter it slightly. Some variability of $K_{a m b}$ is to be expected if the composition of different meteors differs. However, a large seasonal variation is not expected. The fact that Southern Hemisphere and Northern Hemisphere data both depend on temperature and not on the month of the year supports this. Additional reasons for variation in $K_{a m b}$ relate to fragmentation of the meteor upon entry into the atmosphere, and the generation of turbulence within the trail.

Temperatures can vary in time due to the passage of gravity waves, and tides. Such variability can also exist as a function of height across the meteor region. Lübken et al. (1999) shows temperature variability of the order of $10 \%$. There is some seasonal dependence, but it is not large, and cannot account for the observed reduction in decay-time correlation coefficients when the temperature is low.

The pressure varies smoothly with height, as described by the hydrostatic equation, so at first consideration it should give rise to perhaps systematic errors, but not random errors. However, in fact, it turns out that the pressure variation does contribute to the scatter, but in an indirect way.

As the temperature decreases, the scale height of the atmosphere decreases. The vertical resolution of the radar is between 2 and $3.5 \mathrm{~km}$ (see below). In the polar summer mesosphere, where the temperature can be as low $130 \mathrm{~K}$, the scale height is $3.8 \mathrm{~km}$. The pressure variation across, say, $2.5 \mathrm{~km}$ under these conditions is about a factor of two (pressure halves for an altitude increase of $2.5 \mathrm{~km}$ ). But in winter, the temperature is more like $220 \mathrm{~K}$, the scale height is $6.5 \mathrm{~km}$, and the pressure variation across $2.5 \mathrm{~km}$ is less (pressure reduces to about $70 \%$ of the original value for a vertical displacement of $2.5 \mathrm{~km}$ ). This change is much less than the summer-time value, and this variation in scale height as a function of temperature turns out to be the main cause of 


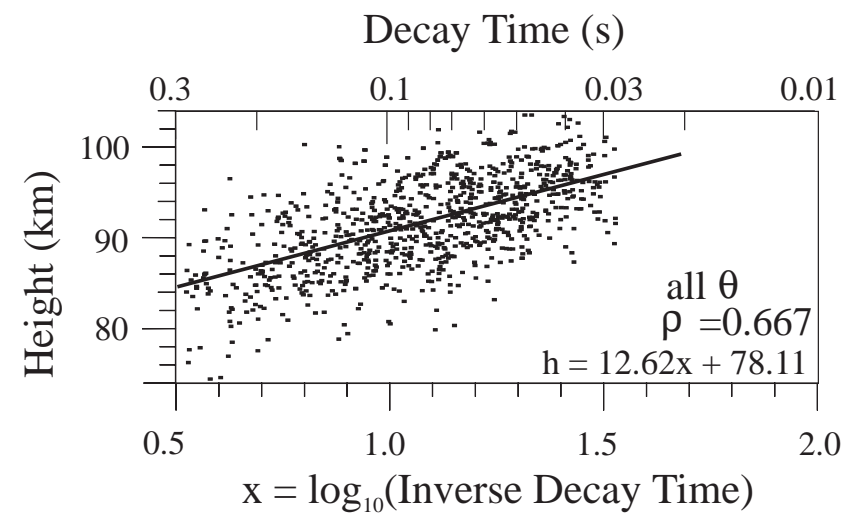

Fig. 3. A graph like that shown in Fig. 1c, but using simulated data and assumed values for errors in $K_{a m b}$, temperature variability and height errors of $27 \%, 8 \%$ and $3.25 \mathrm{~km}$, respectively.

the relationship between the temperature and decay-time correlation coefficient. Although the pressure varies smoothly with height, there is a random element to the determination of height which depends on the radar pulse length, the angular resolution of the interferometer and the size of the first Fresnel zone at the meteor height. It is the pressure variability associated with this height uncertainty which is the main cause of the scatter in graphs like those shown in Fig. 1, and this also explains why the decay-time correlation coefficient is a function of temperature - lower temperatures correspond to smaller scale heights and so a larger change in pressure within the radar resolution.

These effects can be demonstrated with a numerical model.

\section{Numerical model}

The effects described in the previous section have been simulated as follows. To begin, a straight line is defined on a graph of height vs. $\log _{10}\left(1 / \tau_{1 / 2}\right)$, with slope defined according to temperature (see the variable $S_{m}$ in Hocking (1999)). A selection of closely-spaced heights is then chosen, and for each height a value of $\tau_{1 / 2}$ is assigned by reading directly off this straight line. Thus, all of the original (height, $\log 10\left(1 / \tau_{1 / 2}\right)$ ) points lie on this line. Then the values of $\log 10\left(1 / \tau_{1 / 2}\right)$ ) are randomized, with the standard deviation defined according to specified values of $\frac{\Delta T}{T}$ and $\frac{\Delta K_{a m b}}{K_{a m b}}$ (Eq. 5). A Gaussian random number generator has been used. With regard to pressure variation, it is known that the pressure is a strong function of height (hydrostatic equation), and random pressure variability in the data comes about as a result of height error. Therefore, a pressure error term has not been specifically added, but the heights have been randomized and a Gaussian distribution of height errors with standard deviation $\Delta z$ has been assumed. $\Delta z$ is determined by both the pulse resolution of the radar (typically $2 \mathrm{~km}$ ) and the angular resolution of the interferometer (typically 1 to $1.5^{\circ}-$ see Jones et al., 1998). Many meteors are detected at elevations of $30^{\circ}$ to $50^{\circ}$ (zenith angles of $40^{\circ}$ to $60^{\circ}$ ), so the angular resolution is in fact the primary contributor to the height resolution. For a pulse length equivalent to $2 \mathrm{~km}$, and a meteor at an altitude of $90 \mathrm{~km}$ at an angle of $50^{\circ}$ from zenith, a vertical error of about $2 \times \cos (50)=1.3 \mathrm{~km}$ exists due to the pulse, and $90.0 / \cos (50) * \delta \theta * \sin (50)$ due to the angular error of the interferometer. If $\delta \theta$ is taken to be $1.5^{\circ}$, then this second term is about $2.8 \mathrm{~km}$. The total vertical error is about $\sqrt{1.3^{2}+2.8^{2}}=3.25 \mathrm{~km}$. Clearly the angular term dominates at these angles. At zenith angles of $30^{\circ}$ and less, the pulse length starts to dominate the error term. In those cases, it can be beneficial to use shorter pulses. At larger angles than $30^{\circ}$ from zenith, the angular resolution dominates and no advantage is to be had by using shorter pulses.

The result of such randomization is shown in Fig. 3. In this case the selected errors were: $\Delta T / T=8 \%, \Delta z=3.25 \mathrm{~km}$, and $\Delta K_{a m b} / K_{a m b}=27 \%$. Note the strong similarity to the lower plot in Fig. 1. The temperature deduced from these data was $202 \mathrm{~K}$, the decay-time correlation coefficient is very similar to that shown in the lower graph in Fig. 1, the best-fit lines are similar, and the general scatter is similar. The only difference is that there is a $3-\mathrm{km}$ height offset between the two data sets. This arises because of differences in the assumed absolute pressure at $70 \mathrm{~km}$ and the true absolute pressure at that height, which arise because the CIRA pressures (Fleming et al., 1988) were assumed in the model. This will not impact application of this theory to realistic situations, since the offset will not impact the amount of variability.

The values of the errors can be varied, and many hundreds of simulations have been performed. A search has been performed to find the combination of $\Delta K_{a m b}$ and $\Delta z$ which gives us decay-time correlation coefficients closest to observed values for both summer and winter at Resolute Bay. Values of $\frac{\Delta T}{T}$ have been chosen according to Lübken (1999). In fact, only a small range of $K_{a m b}$ and height variances can give us decay-time correlation coefficients which are comparable to measured values, and one such example was shown in Fig. 3. These values are $\Delta K_{a m b} / K_{a m b}=.27+/-.05$ and $\Delta z=3.25 \pm 0.25 \mathrm{~km}$. The value of $\Delta z$ is reasonable, and arises because most meteors are detected at angles of 40 to $60^{\circ}$ from zenith. The reason for an expected value of $3.25 \mathrm{~km}$ was discussed earlier, but this was not assumed in the simulations, and values between 1.5 and $4 \mathrm{~km}$ have been tried. The fact that the derived value was indeed $3.25 \mathrm{~km}$ shows that our understanding of the causes of the scatter is quite accurate. Values of $\Delta K / K$ between $0 \%$ and $100 \%$ were used in the simulations, but the value of $27 \%$ was the only one which correctly matched the observed correlation coefficients for both summer and winter at Resolute Bay. This is an important result because it describes the variability of $K_{a m b}$. Variability of $K_{a m b}$ can occur due to several factors, including fragmentation of the incoming meteoroid, anisotropy in the diffusion rate, plasma instabilities and also the metallic and alkali content of the meteoroid itself (see Jones and Jones, 1990). Our estimate of the variability of $K_{a m b}$ places limits on the combined effects of these processes. Obviously the variability of each such process must independently be less 


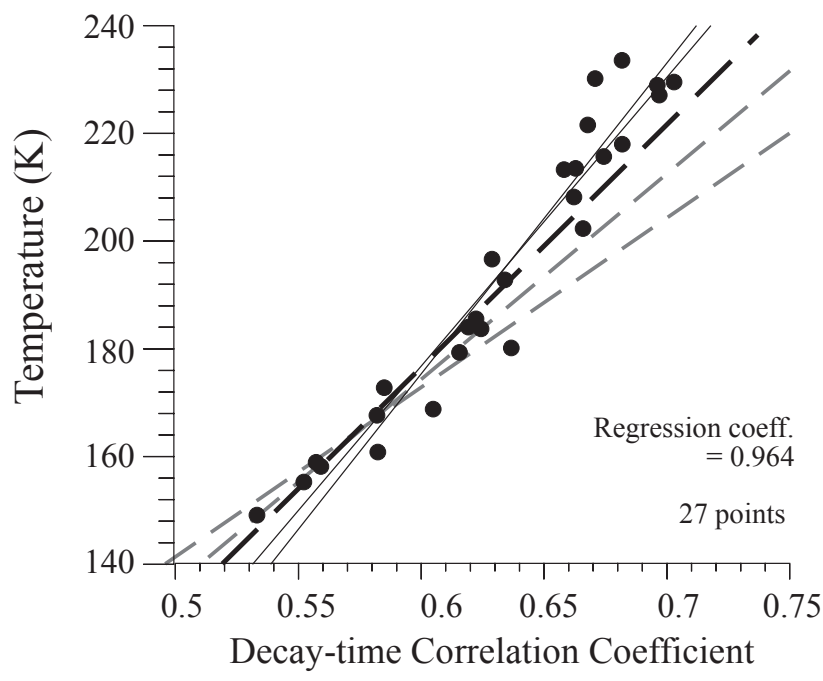

Fig. 4. The points represent the decay-time correlation coefficients deduced from our numerical model for various assumed temperatures (ordinate), using different assumed random number seeds and different assumed initial temperatures. The two thin solid lines represent best line fits to the data, assuming zero error in the temperature (steepest line) and then zero error in the correlation coefficient (shallower line). The broken lines represent experimental fits from Fig. 2.

than $27 \%$, and so this measurement clearly limits the variability of the metallic content of meteoroids as well.

Having produced optimized values for $\Delta K / K, \Delta T / T$ and $\Delta z$, the simulations were rerun for many different assumed temperatures, using many different seeds for the random number generators, but maintaining fixed values for $\Delta K / K$, $\Delta T / T$ and $\Delta z$. It was therefore possible to produce a graph of temperature vs. decay-time correlation coefficient. The result is shown in Fig. 4. Best-fit lines for the regression of the ordinate on the abscissa (shallower solid line) and the abscissa on the ordinate (steeper solid line), are also shown. This graph should be compared to the experimental data in Fig. 2. The best-fit lines from Fig. 2 are overlain on Fig. 4 as broken lines for reference. The steepest broken line is the regression of the abscissa on the ordinate from Fig. 2. The middle broken line assumes equal relative errors in each coordinate, and the shallowest assumes that the decay-time correlation coefficients have zero error and the temperature contains all the error.

The comparison between the theoretical and experimental best-fit lines is quite reasonable, although the experimental case is somewhat shallower in slope. Although agreement is not perfect, it is very close, demonstrating that many of the reasons for the observed scatter of the decay times as a function of height have been properly represented. Differences may arise because of the interannual variability of $\Delta T / T$ and $\Delta K / K$, which has not been considered in this simple model. In addition, the error in height has not been allowed to be a function of zenith angle, and if this were allowed it could represent a minor improvement to the model. How- ever, since the majority of meteors fall in the zenith angles between 40 and $60^{\circ}$, this refinement should not substantially alter the results presented so far.

This graph opens the possibility for an alternative method to use meteor decay times to determine temperatures. Providing that the radar receiver phases are properly calibrated, so that the height determinations have minimal random error, the correlation coefficient between the log of the inverse decay time and height can be used, together with Fig. 4, to deduce the temperature. Based on a comparison of both experimental and simulated data, the best relation to use is $T=360 \rho-42,(T<190 \mathrm{~K})$, where $\rho$ is the decay-time correlation coefficient. The expression works reasonably well for cooler temperatures, but at temperatures above about $190 \mathrm{~K}$ starts to have larger errors associated with it. This is because at lower temperatures the pressure variation with height is most rapid, and so this is the dominant cause of the scatter. However, at higher temperatures, the pressure variation is less, and factors like variability of $K_{a m b}$ and $T$ begin to have a relatively more substantial effect on the correlation. These latter parameters are harder to incorporate into the model since they vary from day to day and between seasons. Finally, it has been assumed that Eq. (3) is accurate. Hocking (1999) has tested the validity of this assumed equation, and shown that it is valid to reasonable order, but variations in exponents of $P$ and $T$ from 1.0 and 2.0, respectively, could still exist (either due to unanticipated geophysical effects, or even errors in the basic theory) and if this were so, it would alter slightly the results of the model.

The method of Hocking (1999) is still suggested as the preferred meteor method to use for determination of temperatures, but it does require knowledge of the mean temperature gradient. This newer "correlation" method has the advantage that it does not depend on a temperature gradient model, and can be useful especially at colder temperatures.

\section{Antenna phase errors}

The above results are therefore consistent with the hypothesis that the observed scatter is largely due to geophysical phenomena. However, there is one other source of error which has not yet been discussed. This has not been considered until now because it is not due to geophysical phenomena, but to instrumental error.

To date it has been assumed that the radar is a "perfect" interferometer. However, when an interferometric radar is established, it is critical that the phase delays through the receivers be properly determined and accounted for. It is also critical that all phase delays between the receiver antennas and associated cabling be properly determined. If these phases are incorrectly measured, errors in angular position determinations can result. If the receiver phases drift in time, and are not properly recorded, similar errors can occur. The phases should be known to within at least $10^{\circ}$ for the SKiYMET class of radars (and accuracies of at least $2^{\circ}$ should be attainable and are highly recommended), and 
phase errors of larger than $25^{\circ}$ can give catastrophic errors in position determination.

In order to complete the analyses of scatter, the effects of random errors in phase calibration on errors in temperature determination have been investigated. To do this, some sample data recorded with the Socorro SKiYMET radar in New Mexico were used. These data were well calibrated. Sample data have been taken from 13,14 and 15 September 2002 and all of the important information for direction determination for each recorded meteor (over 9000 detections) was recorded. Artificial phase errors have then been deliberately introduced into the software, which altered the assumed phase delays of the receivers, and subsequently altered the determination of all meteor positions. Phase offsets were varied between -30 and $30^{\circ}$, and scatter plots like those in Figs. 1 and 3 were produced. The decay-time correlation coefficients produced in this way varied between 0.68 and 0.45 , depending on how far removed the artificial phase offsets were from the true values.

In earlier discussions it was indicated that when the radar is properly calibrated, choosing meteors from different zenith angle bands in the sky altered the decay-time correlation coefficient but did not substantially alter the temperatures derived by the "slope" method (Hocking 1999). However, when the phases are badly calibrated, the "temperature" deduced by the standard analysis does indeed vary, demonstrating in this case that a badly calibrated radar can produce erroneous meteor temperatures. This is therefore an entirely different situation to that discussed in the previous section, where temperature variations caused a decrease in the correlation coefficient. In the case of a badly calibrated system, poorer correlation affects the estimated temperature.

It is therefore of absolutely crucial importance that an interferometric meteor radar be carefully calibrated. All meteor radars used in this paper have been so calibrated, and so are not affected by the above comments, but the simulations should serve as a warning to any users of such meteor radars of the crucial importance of phase calibrations. Phase calibrations should also be continuously monitored throughout the life of the radar.

Figure 5 shows a plot of the "derived temperature" as a function of decay-time correlation coefficient, where the true temperature should be about $200 \mathrm{~K}$ (optimum correlation coefficient $=0.68$ ). Best fit lines are again drawn. Note that the slopes of these lines are very different (differing by over a factor of 2) compared to those in Fig. 4, because the cause of the decorrelation is quite different in this case. Figure 5 could be used to correct the temperatures derived from a poorly calibrated system, provided that the error in the correlation due to the bad phase calibration is known, but this procedure is not normally recommended unless the adjustments are only modest. An error in $\rho$ of $\Delta \rho$ has been found to require a correction to the temperature of $173 \Delta \rho$. As a rule, it is however far more effective to ensure that the radar phases are properly calibrated in the first place rather than to rely on such corrections.

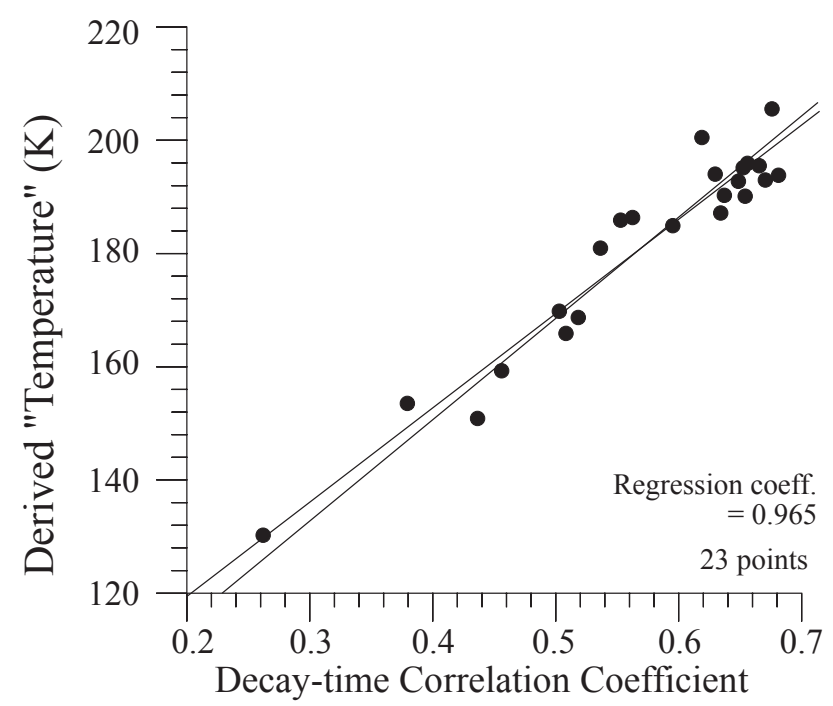

Fig. 5. Graph showing the impact of poor phase calibration on temperatures deduced with an interferometric meteor radar. It has been assumed that the temperature is deduced by the "slope method" (Hocking, 1999). Poor phase calibration leads to reduced correlation coefficients, which, in turn, produces artificially low temperatures.

\section{Effects of magnetic and electric fields}

Above about $93 \mathrm{~km}$ collision frequencies are small enough that electric and magnetic fields can cause anisotropic diffusion. The most recent theoretical studies of this effect are due to Robson (2001). Other related references include Jones (1991), Ceplecha et al. (1998) and Elford and Elford (2001), and all generally agree on a transition height of about $93 \mathrm{~km}$ for the transition from isotropic to anisotropic diffusion. In this section we will examine the impact of ionospheric and plasma effects on variability of $\tau_{1 / 2}$.

Robson (2001) gives the following expressions for the diffusion coefficient for a meteor trail:

$D_{e f f}=D_{\|} \sin ^{2} \mu \sin ^{2} \theta+D_{\perp}\left(1-\sin ^{2} \mu \sin ^{2} \theta\right)$.

This can also be written (see Robson, 2001, Eq. 9) as

$D_{e f f}=D_{\|} \cos ^{2} \alpha+D_{\perp}\left(1-\cos ^{2} \alpha\right)=D_{\|} \cos ^{2} \alpha+D_{\perp} \sin ^{2} \alpha$,

where $\alpha$ is simply the angle between the viewing direction (which is perpendicular to the meteor trail) and the magnetic field lines.

Robson also relates the parallel and perpendicular diffusion coefficients by the expression

$D_{\|}=D_{\perp}(1+\zeta)$,

where $\zeta=\frac{e^{2} B^{2}}{m_{e} \nu_{e} \mu_{i} \nu_{i}}$, and $m_{e}$ is the electron mass, $\mu_{i}$ is the reduced mass of the neutral-ion pair involved in the collisions during diffusion, $v_{e}$ is the electron collision frequency and $v_{i}$ is the ion collision frequency with the neutrals. (Robson used $\rho$ for our symbol $\zeta$, but we have used $\zeta$ to avoid confusion with our earlier use of $\rho$.) 

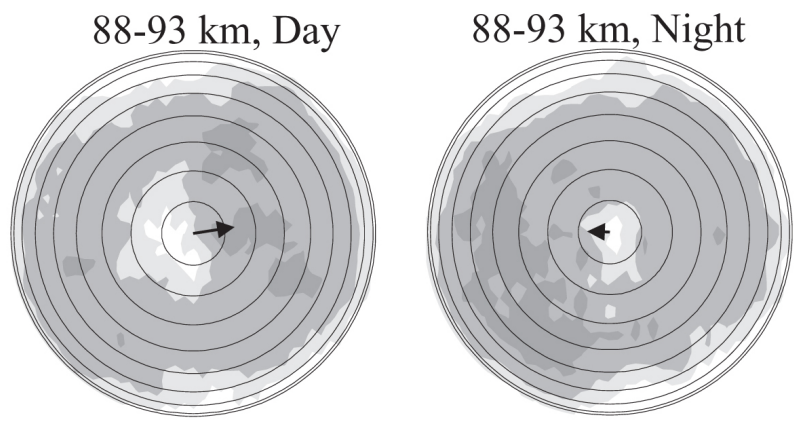

94-100 km, Day

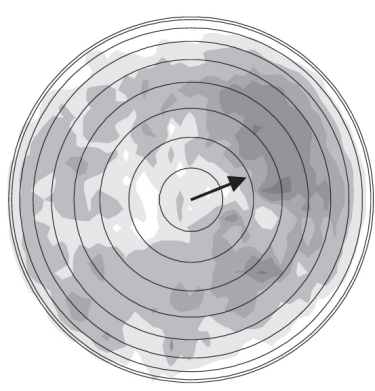

94-100 km, Night

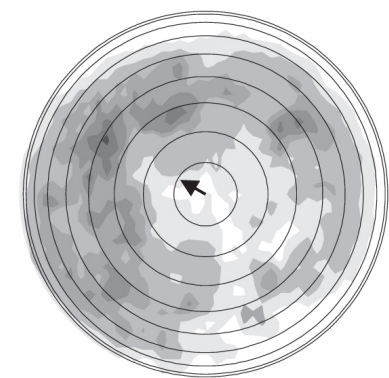

$1 / \tau_{1 / 2}$

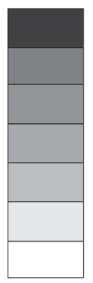

$>24.0$

$21.0-24.0$

$18.0-21.0$

$15.0-18.0$

$9.0-15.0$

$3.0-9.0$

$<3.0$

Fig. 6. Contour map of the average inverse decay times as a function of zenith angle and azimuth for the Clovar radar, broken up according to daytime and nightime, for the period from June to August 1999 . North is vertical, and the black circles show $10^{\circ}$ steps in zenith angle.

We can determine the height at which anisotropy becomes important by finding when $1+\zeta$ departs substantially from unity. In practice this is complicated by the fact that it is not possible to use standard "hard-sphere" approximations for collision cross sections (collision cross sections are energy dependent), but more proper cross sections have been deduced using experimental laboratory data (e.g. Elford and Elford, 2001). At $95-96 \mathrm{~km}$ altitude, $\frac{D_{\|}}{D_{\perp}}$ is of the order of 10 , while at about $93 \mathrm{~km}, \frac{D_{\|}}{D_{\perp}}$ is of the order of 2 . At lower heights, this ratio quickly approaches unity. Hence, anisotropy effects might be expected to be important above typically $93 \mathrm{~km}$, according to this theory. As a result, we would expect to see different meteor-trail radar lifetimes depending on the angle at which the trail is seen by the radar relative to the magnetic field. A lesser (or no) effect should be evident at heights below this altitude.

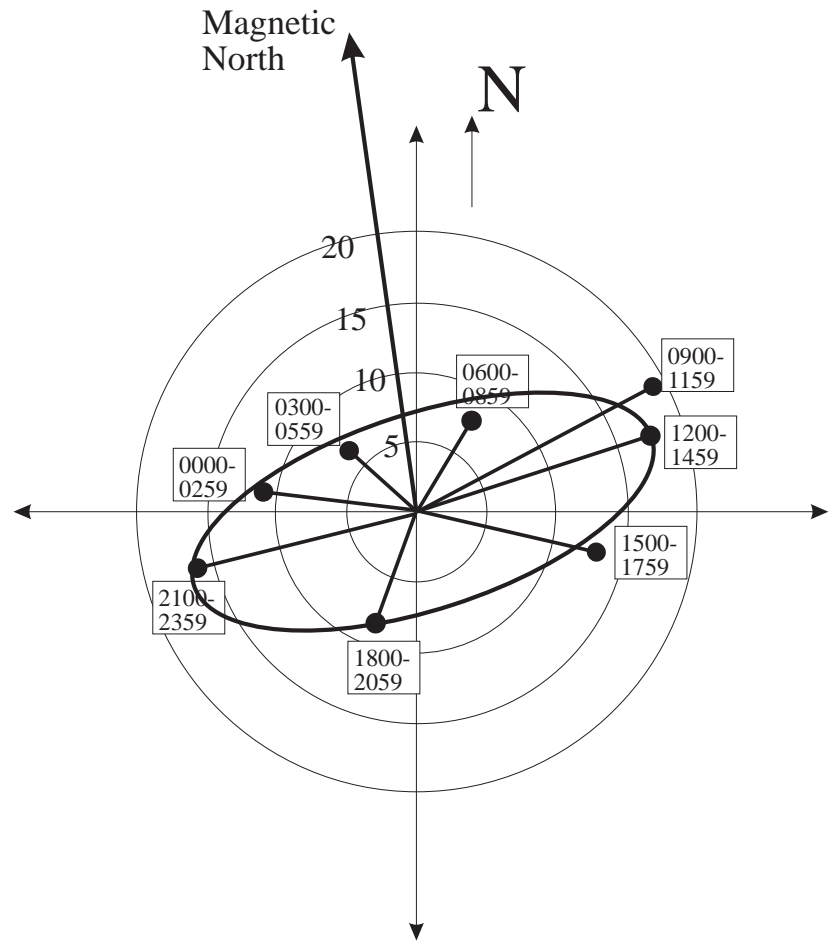

Fig. 7. Average inverse decay vectors plotted as a function of time of day for the summer of 1999.

This possibility has been investigated by plotting contours of typical mean inverse decay times as a function of zenith and azimuth. An example for the Clovar radar, using data from all of summer in 1999, is shown in Fig. 6. We have divided the data into the height ranges (i) 88 to $93 \mathrm{~km}$, and (ii) greater than $93 \mathrm{~km}$. These have been plotted on separate graphs. In each height range we have divided the meteors into bins with inverse decay times in the intervals specified by the grey-scale box in the figure. These data sets have then been further subdivided into (zenith, azimuth) bins of $\operatorname{size}(\Delta[\sin (\theta) \cos (\phi)], \Delta[\sin (\theta) \cos (\phi)])=(0.05,0.05)$ (where $\theta$ is the zenithal angle and $\phi$ is the azimuthal angle), and the numbers of meteors in each bin have been counted. A $3 \times 3$ box-car running mean was then applied to introduce some smoothing. In general, there are no bins with zero counts - the total number of meteors used exceeds 50000 . Except for the region immediately overhead (from which few meteors are detected), typical mean decay time values $\left(<\frac{1}{\tau_{1 / 2}}>\right)$ in the height range 88 to $93 \mathrm{~km}$ are of the order of 9 to 15 , but with occasional excursions to 15-18. The overall typical variation is less than $25 \%$. However, in the height region above $93 \mathrm{~km}$, there is much more structure, with mean values rising as high as $24 s^{-1}$ and falling as low as 9. This is consistent with the above theoretical arguments.

However, it can be seen that the region of general maximum values of $<\frac{1}{\tau_{1 / 2}}>$ is different during night and day. This is not consistent with previously published theories, and suggests that the diffusion anisotropy also depends on factors other than the magnetic field strength and orientation. 
In order to investigate this further, a vector has been calculated with the components $\left(<\frac{1}{\tau} \cos (\phi)>,<\frac{1}{\tau} \sin (\phi)>\right)$, where $\phi$ is the azimuthal angle of each meteor location and $\tau$ is the decay time. Such an average produces a vector which points in the general direction of the larger values of $1 / \tau$, or direction of fastest diffusion. Examples are shown in Fig. 6.

The data were then broken into 3-h clusters, according to time of day, and plotted as in Fig. 6, for the data above $93 \mathrm{~km}$. In general, decreasing the cluster time interval enhanced the contrast between maximum and minimum values of $\left\langle\frac{1}{\tau}\right\rangle$, and produced more concentrated regions of maxima. When the vectors produced by these plots were combined, Fig. 7 resulted. This result is for the Clovar radar, but similar results existed at other sites. The position of maximum diffusion clearly changes with time along a quasi-elliptical path. An ellipse has been fitted to the points. The ellipse is rotated $18^{\circ}$ north of east, while magnetic north is $8^{\circ}$ west of north at London, Ont. Clearly there is a diurnal rotation, suggesting a tidal influence, probably via an $\boldsymbol{E} \times \boldsymbol{B}$ effect. It seems clear that the analyses by Robson (2001) and authors before him need to be considerably improved, since the hypothesis that the diffusion coefficient anisotropy is a simple function of the magnetic field is inadequate. Further analyses are beyond the scope of this paper.

However, it is clear, at least experimentally (Figs. 6 and 7) that there will be some natural varability in the decay times, dependent on the angular location of the meteors and time of day, and this will naturally contribute to the overall scatter. However, any contribution to enhanced variability of decay times due to plasma effects will exist primarily above 92-93 km altitude, and below this height the effect is generally minimal. Only $8 \%$ of our meteors are detected at these upper heights, so our preceding conclusions should only be marginally affected by plasma processes. Interestingly, it will create an interesting bias in that the increase in scatter will not be uniform but will be biased to greater heights. Nevertheless, Fig. 6 suggests that even below $93 \mathrm{~km}$ there is some variation as a function of angle, so it would be true that at least some part of the $27 \%$ variability in $K_{a m b}$ can be attributed to these plasma effects. The actual fraction is unclear at this time.

\section{Conclusions}

When $\log$ (inverse decay times) determined by meteor radar are plotted as a function of height, the points show considerable scatter. Statistical fluctuation is least for meteors close to overhead, and greatest for meteors at low elevations. The correlation is greatest when atmospheric temperatures are high, and reduced at low temperatures. The causes of this scatter have been investigated, and have been found to be due to (i) intrinsic height error, (ii) variations in instantaneous temperature and (iii) variations in $K_{a m b}$. Of these, the first is the most important. Due to limits associated primarily with angular resolution, the effective vertical resolution is about $3 \mathrm{~km}$ for many of the meteors, since the bulk lie at angles of $40^{\circ}$ to $60^{\circ}$ from zenith. This height resolution, coupled with the fact that the pressure changes significantly over one scale height, produces the largest variability of the decay times.

By developing a computer model which simulates the causes of errors in height and decay times, it has been possible to simulate the observed scatter plots moderately well, both for summer and winter time conditions. By optimizing the experimental fits, it has been shown that the quantity $K_{a m b}$ has an intrinsic typical variability from meteor to meteor of about $27 \%$, and this is the first time that the variability in this parameter has been measured. It should be emphasized that this is a "typical" value, and substantial alteration of the mean and standard deviation during major showers and even as a function of time of year cannot be ruled out. Indeed, limited intraannual variability of this parameter might account partly for the fact that perfect comparison with experimental data was not achievable. Variability of $K_{a m b}$ can occur due to fragmentation of the incoming meteoroid, anisotropy in the diffusion rate, plasma instabilities and variations of the metallic and alkali content of the meteoroid itself.

It has been demonstrated that, if carefully employed, the correlation coefficient of $\log$ (inverse decay time) versus height can be used as a proxy for temperature, and is especially useful at lower temperatures. This parameter does not require knowledge of the mean temperature gradient, an advantage over the more common method (Hocking, 1999). At lower temperatures the relative contribution from the height uncertainty (and hence the pressure variation) is greatest, since the scale height is smaller, resulting in more variation in decay time over a fixed height range. Hence, at lower temperatures variability in temperature and $K_{a m b}$ are relatively less dominant, and the correlation is principally defined by the scale height (and hence by the temperature). At high temperatures (especially greater than $200 \mathrm{~K}$ ), variability in $T$ and $K_{a m b}$ becomes relatively more important, and somewhat destroys the simple relation between correlation coefficient and temperature.

Finally, the crucial importance of calibrating the receiver phases of the interferometer has been discussed, and simulations demonstrating the impact of errors in these calibrations have been performed.

Acknowledgements. The Natural Sciences and Engineering Research Council of Canada is acknowledged for partial support of this research program. It also provided funds for the Resolute Bay radar. SRI and the National Science Foundation also provide support for the Resolute Bay facility. The SKiYMET radar at Socorro is wholly owned by Mardoc Inc., and leased to the University of Western Ontario. B. Fuller, of Genesis Software Inc., is thanked for providing data from the Delamere and Learmonth SKiYMET sites in Australia. Thanks also to P. Krehbiel, A. Erickson, N. Euart and A. Tubesing for support of the SKIYMET radar at Socorro. Thanks also to P. T. Jayachandran for his insightful suggestions in regard to analysis of the decay times above $93 \mathrm{~km}$.

Topical Editor U.-P. Hoppe thanks L. Dyrud and another referee for their help in evaluating this paper. 


\section{References}

Ceplecha, Z., Borovicka, J., Elford, W. G., ReVelle, D. O., Hawkes, R. L., Porubcan, V., and Simek, M.: Meteor phenomenoa and bodies, Space Sci. Rev., 84, 327-471, 1998.

Cervera, M. A. and Reid, I. M.: Comparison of atmospheric parameters derived from meteor observations with CIRA Radio Sci., 35, 833-843, 2000.

Chilson P. B., Czechowsky, P., and Schmidt, G.: A comparison of ambipolar diffusion coefficients in meteor trains using VHF radar and UV lidar, Geophys. Res. Lett., 23, 2745-1748, 1996.

Dyrud, L. P., Oppenheim, M. M., and vom Endt, A.F.: The anomalous diffusion of meteor trails, Geophys. Res. Lett. , 28, 27752779, 2001

Dyrud, L. P., Oppenheim, M. M., Close, S. and Hunt, S.: Interpretation of non-specular radar meteor trails, Geophys. Res. Letts., vol. 29, no. 21, 2012, doi:10.1029/2002GL015953, 2002.

Elford, W. G. and Elford, M. T.: The effective diffusion coefficient of meteor trails above $100 \mathrm{~km}$, in:Proceedings of the Meteoroids 2001 Conference, 6-10 August 2001, Kiruna, Sweden, edited by: Warmbein, B., ESA SP-495, Noordwijk: ESA Publications Division, ISBN 92-9092-805-0, 357-359, 2001.

Fleming, E. L., Chandra, S., Schoeberl, M. R., and Barnett, J. J.: Monthly mean global climatology of temperature, wind, geopotential height and pressure from $0-120 \mathrm{~km}$, NASA Technical Memorandum 100697, 85, 1988.

Hocking, W. K., Thayaparan, T. and Jones, J.: Meteor decay times and their use in determining a diagnostic mesospheric temperature-pressure parameter: methodology and one year of data, Geophys. Res. Letts., 24, 2977-2980, 1997.

Hocking, W. K.: Temperatures using radar-meteor decay times, Geophys. Res. Lett., 26, 3297-3300, 1999.

Hocking, W. K., Fuller, B. and Vandepeer, B.: Real-time Determination of Meteor-related Parameters Utilizing Modern Digital Technology, J. Atmos. Solar-Terr. Physics, 63, 155-169, 2001a.

Hocking, W. K., Thayaparan, T., and Franke, S. J.: Method for statistical comparison of geophysical data by multiple instruments which have differing accuracies, Adv. Space Res., 27, numbers 6-7, 1089-1098, 2001b.
Hocking, W. K., Kelley, M. C., Rogers, R., Brown, W. O. J., Moorcroft, D. and St. Maurice, J.-P.: Resolute Bay VHF radar: A multi-purpose tool for studies of tropospheric motions, middle atmosphere dynamics, meteor physics and ionospheric physics, Radio Sci., 36, 1839-1857, 2001c.

Hocking, W. K. and Hocking, A.: Temperature tides determined with meteor radar, Ann. Geophys., 20, 1447-1467, 2002.

Jones, J.: On the decay of underdense radio meteor echoes, Mon. Not. Roy. Astron, Soc., 173, 637-647, 1975.

Jones, W. and Jones, J.: Ionic diffusion in meteor trails, J. Atmos. Terr. Phys., 52, 185-191, 1990.

Jones, W.: Theory of diffusion of meteor trains in the geomagnetic field, Planet. Space Sci., 39, 1283-1288, 1991.

Jones, J., Webster, A. R., and Hocking, W. K.: An improved interferometer design for use with meteor radars, Radio Sci., 33, 55-65, 1998.

Kaiser, T. R., Pickering, W. M., and Watkins, C. D.: Planetary and Space Science, 17, 519, 1968.

Liu, A. Z., Hocking, W. K., Franke, S. J., and Thayaparan, T.: Comparsion of $\mathrm{Na}$ Lidar and meteor radar wind measurements at Starfire Optical Range, NM, USA, J. Atmos. Ter. Phys., 64, 31-40, 2002.

Lübken, F.-J. and Von Zahn, U.: Thermal structure of the mesopause region at polar latitudes, J. Geophys. Res., 96, 20 841-20 857, 1991.

Lübken, F.-J.: Thermal structure of the Arctic summer mesosphere, J. Geophys. Res., 104, 9135-9149, 1999.

Oppenheim, M. M., Vom Endt, A. F., and Dyrud, L. P.: Electrodynamics of meteor trail evolution in the equatorial E-region ionosphere,Geophys. Res. Lett., 27, 3173-3176, 2000.

Robson, R. E.: Dispersion of meteor trails in the geomagnetic field, Phys. Rev. E, 63, 026404-1 to 026404-5, 2001.

Tsutsumi, M., Tsuda, T. and Nakamura, T.: Temperature fluctuations near the mesopause inferred from meteor observations with the middle and upper atmosphere radar, Radio Sci., 29, 599-610, 1994. 